\title{
Mathematical formulations of Hebbian learning
}

\author{
Wulfram Gerstner ${ }^{1}$, Werner M. Kistler ${ }^{2}$ \\ ${ }^{1}$ Swiss Federal Institute of Technology Lausanne, Laboratory of Computational Neuroscience, EPFL-LCN, \\ 1015 Lausanne EPFL, Switzerland \\ ${ }^{2}$ Department of Neuroscience, Erasmus University, Rotterdam, The Netherlands
}

Received: 1 February 2002 / Accepted: 28 March 2002

\begin{abstract}
Several formulations of correlation-based Hebbian learning are reviewed. On the presynaptic side, activity is described either by a firing rate or by presynaptic spike arrival. The state of the postsynaptic neuron can be described by its membrane potential, its firing rate, or the timing of backpropagating action potentials (BPAPs). It is shown that all of the above formulations can be derived from the point of view of an expansion. In the absence of BPAPs, it is natural to correlate presynaptic spikes with the postsynaptic membrane potential. Time windows of spike-time-dependent plasticity arise naturally if the timing of postsynaptic spikes is available at the site of the synapse, as is the case in the presence of BPAPs. With an appropriate choice of parameters, Hebbian synaptic plasticity has intrinsic normalization properties that stabilizes postsynaptic firing rates and leads to subtractive weight normalization.
\end{abstract}

\section{Introduction}

Over the last 50 years, a large body of experimental and theoretical work on synaptic plasticity and learning has been inspired by Hebb's postulate: "When an axon of cell A is near enough to excite cell B or repeatedly or persistently takes part in firing it, some growth process or metabolic change takes place in one or both cells such that A's efficiency, as one of the cells firing B, is increased" (Hebb 1949). Today this famous postulate is often rephrased in the sense that modifications in the synaptic transmission efficacy are driven by the correlations in the firing activity of the pre- and the postsynaptic neuron. Even though the idea of learning through correlations dates further back in the past (James 1890),

Correspondence to: W. Gerstner (e-mail: wulfram.gerstner@epfl.ch,

Tel.: +41-21-6936713, Fax: +41-21-6935263) correlation-based learning rules are now generally called Hebbian learning.

Hebb formulated his principle on purely theoretical grounds. He realized that such a principle would help to stabilize specific neuronal activity patterns in the brain. If neuronal activity patterns correspond to behavior, then stabilization of specific patterns implies learning of specific behaviors (Hebb 1949). Even though Hebb's statement was essentially a theoretical one, he did not formulate it himself in mathematical terms. In this paper we review several mathematical formulations of Hebbian learning. We start in Sect. 2 with rate-based descriptions and turn then in Sect. 3 to spike-based models of Hebbian plasticity. In both cases we focus on a single synapse $w_{i j}$ that transmits signals from a presynaptic neuron $j$ to a postsynaptic neuron $i$ (Fig. 1). Large portions of the text of the present paper are based on a recent book on spiking neuron models (Gerstner and Kistler 2002).

\section{Rate-based Hebbian learning}

In rate-based neuron models, the activity of a given neuron $i$ is described by its firing rate $v_{i}$ which is related to the membrane potential $u_{i}$ by a nonlinear monotonically increasing function $g$ :

$v_{i}=g\left(u_{i}\right)$.

The membrane potential in turn can be calculated from the presynaptic firing rates $v_{j}$ and the synaptic weights $w_{i j}$, i.e., $u_{i}=\sum_{j} w_{i j} v_{j}$. In the following we assume that the firing rates $v_{i}$ and $v_{j}$ of the pre- and the postsynaptic neuron are constant during one trial of an experiment. For several classical experiments on long-term potentiation (LTP) this is a reasonable assumption (for reviews see Brown et al. 1989; Bliss and Collingridge 1993). LTP can, for example, be introduced by high-frequency trains of presynaptic pulses at several synapses that are maintained over a time $\mathscr{T}$. In such a situation the temporal resolution is rather coarse, and a description of pre- and postsynaptic activity by fixed rates is appro- 


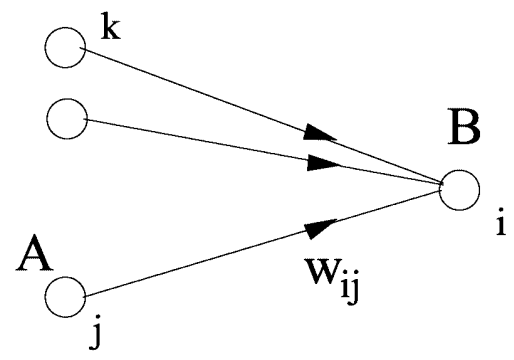

Fig. 1. The change at synapse $w_{i j}$ depends on the states of the presynaptic neuron $j$ and the postsynaptic neuron $i$, and the present efficacy $w_{i j}$, but not on the state of other neurons $k$

priate. Time-dependent stimulation paradigms will be treated in the context of the spike-based formulation outlined in Sect. 3.

We consider the weight change $\Delta w_{i j}$ during one learning trial. Since the total weight change during a trial depends on the duration of the trial, we focus on the rate of change $\mathrm{d} w_{i j} / \mathrm{d} t=\Delta w_{i j} / \mathscr{T}$.

\subsection{Model requirements}

According to Hebb's postulate, the weight should increase if, during an experimental trial, both neurons are active together. Can we give a more precise mathematical description of Hebb's ideas? Our aim is to formulate the weight change during Hebbian learning as a function of the activity of the pre- and the postsynaptic neuron. From our point of view, at least six aspects are important for the formulation of a useful plasticity model, as described in Sects. 2.1.1-2.1.6.

2.1.1 Locality. The learning rule for the synapse $w_{i j}$ connecting neuron $j$ to neuron $i$ should depend only on the activity of $j$ and $i$ and not on the state of other neurons $k \neq i, j$. In a rate model the state of neuron $i$ is fully characterized by its firing rate $v_{i}$. Given $v_{i}$, the membrane potential $u_{i}$ follows from (1), i.e., $u_{i}=g^{-1}\left(v_{i}\right)$, where $g^{-1}$ denotes the inverse of the transfer function $g$. The only other variable in a rate model that is locally available at the synapse is the present value $w_{i j}$ of the synaptic efficacy itself. Mathematically, we may therefore write

$\frac{\mathrm{d}}{\mathrm{d} t} w_{i j}=F\left(w_{i j} ; v_{i}, v_{j}\right)$

where $F$ is a yet-unknown function (Sejnowski and Tesauro 1989; Brown et al. 1991; Kohonen 1984). Hebb's formulation is clearly consistent with the locality requirement.

2.1.2 Cooperativity. Hebb's formulation 'takes part in firing it' implies that both the pre- and the postsynaptic neuron must be active to induce a weight increase. It furthermore suggests a causal relationship between the firings. We will return to an implementation of causality in Sect. 3. At the moment we restrict ourselves to the requirement of simultaneous activity of the pre- and the postsynaptic neuron. How can we implement this information in the function $F$ in (2)? Since $F$ is a function of the rates $v_{i}$ and $v_{j}$, we may expand $F$ about $v_{i}=v_{j}=0$. An expansion to second order in the rates yields

$$
\begin{aligned}
\frac{\mathrm{d}}{\mathrm{d} t} w_{i j} \approx & c_{2}^{\text {corr }}\left(w_{i j}\right) v_{i} v_{j}+c_{2}^{\text {post }}\left(w_{i j}\right) v_{i}^{2}+c_{2}^{\text {pre }}\left(w_{i j}\right) v_{j}^{2} \\
& +c_{1}^{\text {pre }}\left(w_{i j}\right) v_{j}+c_{1}^{\text {post }}\left(w_{i j}\right) v_{i}+c_{0}\left(w_{i j}\right) \\
& +\mathcal{O}(v) .
\end{aligned}
$$

The first term on the right-hand side of (3) picks up the correlations between pre- and postsynaptic activity. In fact, it is this bilinear term $v_{i} v_{j}$ - namely, a term that is sensitive to the correlations between pre- and postsynaptic activity - which makes Hebbian learning a useful concept. The simplest implementation of Hebbian plasticity would be to require $c_{2}^{\text {corr }}>0$ and set all other parameters in the expansion (3) to zero:

$\frac{\mathrm{d}}{\mathrm{d} t} w_{i j}=c_{2}^{\mathrm{corr}}\left(w_{i j}\right) v_{i} v_{j}$.

Equation (4) with fixed parameter $c_{2}^{\text {corr }}>0$ is the prototype of Hebbian learning. A learning rule with $c_{2}^{\text {corr }}<0$ is usually called anti-Hebbian. We note that, if we continue the expansion on the right-hand side of (3), more and more complex learning rules can be constructed. The next terms would be of order $v^{3}$ : terms of the form $v_{i} v_{j}^{2}, v_{j} v_{i}^{2}$, etc.

2.1.3 Synaptic depression. Hebb's original proposal gives no rule for a decrease of synaptic weights, but only refers to the conditions under which a strengthening of synapses should occur. It is clear, however, that a system where synapses can only increase and never decrease is bound to be useless. An option for decreasing the weights (synaptic depression) is therefore a necessary requirement for any useful learning rule. This can be achieved, for example, by a weight decay, i.e., we take the parameter $c_{0}$ in (3) as

$c_{0}\left(w_{i j}\right)=-\gamma_{0} w_{i j}$

with $\gamma_{0}>0$. Synaptic depression can also be implemented by several other combinations of the factors $c_{1}^{\text {post }}, c_{1}^{\text {pre }}$, and $c_{0}$. For example, the rule $\frac{\mathrm{d}}{\mathrm{d} t} w_{i j}=\left(v_{i}-v_{\theta}\right) v_{j}$ could be implemented by the choice $c_{2}^{\text {corr }}=1, c_{1}^{\text {pre }}=-v_{\theta}<0$, and all other parameters equal to zero. Such a rule is called presynaptically gated, since presynaptic activity is a necessary requirement for any change; the activity level of the postsynaptic neuron determines the direction of the change. An overview of various possibilities of implementing synaptic depression in the framework of (3) is given in Table 1.

2.1.4 Boundedness. In realistic rules, weights at excitatory synapses should remain bounded in a range $0 \leq w_{i j} \leq w^{\max }$, where $w^{\max }$ is the maximal weight value that is sustainable by the biochemical machinery implementing the synaptic connection. To achieve 
Table 1. The change $\frac{\mathrm{d}}{\mathrm{d} t} w_{i j}$ of a synapse from $j$ to $i$ for various Hebb rules as a function of pre- and postsynaptic activity. $O N$ indicates a neuron firing at maximal rate $\left(v=v^{\max }\right)$, whereas $O F F$ means an inactive neuron $(v=0)$. From left to right: standard Hebb rule,

\begin{tabular}{lllllll}
\hline Post & Pre & $\frac{\mathrm{d}}{\mathrm{d} t} w_{i j} \propto$ & $\frac{\mathrm{d}}{\mathrm{d} t} w_{i j} \propto$ & $\frac{\mathrm{d}}{\mathrm{d} t} w_{i j} \propto$ & $\frac{\mathrm{d}}{\mathrm{d} t} w_{i j} \propto$ & \\
$i$ & $j$ & $v_{i} v_{j}$ & $v_{i} v_{j}-c_{0}$ & $\left(v_{i}-v_{\theta}\right) v_{j}$ & $v_{i}\left(v_{j}-v_{\theta}\right)$ & $\begin{array}{c}\mathrm{d} \\
\mathrm{d} t\end{array} w_{i j} \propto$ \\
\hline ON & ON & + & + & + & + & + \\
ON & OFF & 0 & - & 0 & - & - \\
OFF & ON & 0 & - & - & 0 & + \\
OFF & OFF & 0 & - & 0 & 0 & + \\
\hline
\end{tabular}

Hebb with decay, presynaptic gating, postsynaptic gating, and covariance rule. The parameters are $0<v_{\theta}<v^{\max }$ and $0<c_{0}<\left(v^{\max }\right)^{2}$. Angular brackets denote an expectation value boundedness, we make use of the dependence of the parameters in (3) upon $w_{i j}$. Since $F$ on the right-hand side of (2) is a function of $w_{i j}$, the expansion coefficients $c_{2}^{\text {corr }}, c_{2}^{\text {post }}, c_{2}^{\text {pre }}, c_{1}^{\text {post }}, c_{1}^{\text {pre }}$, and $c_{0}$ will also depend on the weight $w_{i j}$. A suitable choice of the $w_{i j}$ dependence of the positive parameters guarantees that the weight cannot become larger than an upper bound $w^{\max }$; similarly, a suitable choice of the negative parameters assures that the $w_{i j}$ cannot decrease below a minimum value of zero (Oja 1982; Kohonen 1984; Miller and MacKay 1994).

In a simple formulation of a 'saturating' weight dependence, we take the parameter $c_{2}^{\text {corr }}$ in (3) as

$c_{2}^{\text {corr }}\left(w_{i j}\right)=\eta_{0}\left(w^{\max }-w_{i j}\right)$

with a constant $\eta_{0}>0$. The factor $\left(w^{\max }-w_{i j}\right)$ can be seen as an implementation of 'soft' bounds. The closer a weight is to its maximum, the smaller the effect of an experimental trial with an LTP-induction protocol. As a modeling alternative to the 'soft' bounds, we can instead use 'hard' bounds: growth of the synaptic weights has a constant factor $c_{2}^{\text {corr }}$ as long as $w_{i j}<w^{\max }$ and stops if $w_{i j}=w^{\max }$. Hence, hard bounds correspond to the replacement $\left(w^{\max }-w_{i j}\right) \longrightarrow \mathscr{H}\left(w^{\max }-w_{i j}\right)$, where $\mathscr{H}(\cdot)$ denotes the Heaviside step function. Similarly, in a hard-bound formulation the weight decrease would simply stop at $w_{i j}=0$, whereas in the soft-bound formulation all negative factors would be taken as proportional to $w_{i j}$.

2.1.5 Competition. A further useful feature of learning rules is competivity. If some weights grow, they do so at the expense of others that must decrease. Ideally, competivity should be a consequence of the learning rule (3) and should not require any additional assumptions. One specific implementation of competivity relies on the normalization of the set of weights $w_{i j}$ of all synapses converging onto the same postsynaptic neuron. While, at a first glance, such a normalization step would seems to violate the requirement of locality, it can in fact be replaced by purely local rules. An example is Oja's rule (Oja, 1982) which is found from (3) if we take $c_{2}^{\text {post }}=-\eta_{0} w_{i j}$, $c_{2}^{\text {corr }}=\eta_{0}>0$, and set all other parameters to zero: $c_{0}=c_{1}^{\text {pre }}=c_{2}^{\text {pre }}=c_{1}^{\text {post }}=0$.

2.1.6 Long-term stability. Most of the learning theories concentrate on the induction of weight changes. Once the 'learning session' is over, weights are taken as fixed parameters. Most neural systems, however, are subject to constantly changing input. If systems continue to remain adaptive, care must be taken that previously learned information is not lost. Grossberg has coined the term 'stability-plasticity dilemma' for this problem (Carpenter and Grossberg 1987; Grossberg 1987). A simple overwriting of previously stored information, sometimes called the 'palimpsest property,' should be avoided.

To approach this problem, Fusi et al. (2000) have studied the problem of the consolidation of weights. They argue that consolidation of previously learned items is possible with a weight dynamics that converges to binary weight values $w_{i j}=0,1$. In our framework, such a dynamics can be implemented by setting

$c_{0}\left(w_{i j}\right)=-\gamma w_{i j}\left(1-w_{i j}\right)\left(w_{\theta}-w_{i j}\right)$

where $0<w_{\theta}<1$ and $\gamma>0$. Small weights $w_{i j}<w_{\theta}$ decay to 0 ; large weights $w_{i j}>w_{\theta}$ increase towards 1 .

If (7) is inserted into (3), then the effects of learning persist (or are even increased) after the end of a learning trial. A combination of (7) with other linear and secondorder terms in the plasticity equation (3) can therefore be considered as a model of consolidation of synaptic plasticity. In most current formulations of synaptic plasticity, the problem of weight consolidation is disregarded.

\subsection{Discussion: relation to other approaches}

While the requirements listed in Sects. 2.1.1-2.1.6 pose a number of constraints for the formulation of learning rules, the framework sketched in (3) is general enough to classify various well-known learning rules. The Bienenstock-Cooper-Monroe (BCM) rule, for example,

$\frac{\mathrm{d}}{\mathrm{d} t} w_{i j}=\eta \phi\left(v_{i}-v_{\theta}\right) v_{j}$

is obtained if we expand the function $F$ in (2) to linear order in the presynaptic rate $v_{j}$ while keeping all higherorder terms in the postsynaptic variable $v_{i}$. The function $\phi$ can in fact be identified with $\mathrm{d} F / \mathrm{d} v_{j}$ evaluated at $v_{j}=0$. In the BCM theory, $\phi$ and $v_{\theta}$ are chosen so that $\phi(0)=0$ and $\phi^{\prime}(0)>0$; that is, $\phi(x)$ has a zero crossing at $x=0$ with positive slope (e.g., $\phi(x)=x-x^{3}+\ldots$ ). It is easy to demonstrate that an output rate $v_{i}=v_{\theta}$ is an unstable fixed point under the dynamics (8). To see this, we simply note that for fixed input rates $v_{j}>0$ and 
monotonic gain function $g$, all weights increase without bounds if $v_{i}>v_{\theta}$. In order to avoid unlimited growth of weights, $v_{\theta}$ is therefore taken in the BCM theory as an adaptive parameter $v_{\theta}=\left\langle v_{i}\right\rangle$, where $\left\langle v_{i}\right\rangle$ is a short-term average of the output rate. The notion of a running short-term average goes beyond the current framework, since it requires some memory, i.e., it is nonlocal in time. We will see, however, that it can be incorporated in the more general framework that is developed in Sect. 3 for spike-based learning rules.

The short-term average plays also a role in the covariance rule of Sejnowski and Tesauro (1989):

$\frac{\mathrm{d}}{\mathrm{dt}} w_{i j}=\left(v_{i}-\left\langle v_{i}\right\rangle\right)\left(v_{j}-\left\langle v_{j}\right\rangle\right)$.

It is based on an expansion of $F$ about a running average of $v_{i}$ and $v_{j}$, rather than on an expansion about zero. For fixed $\left\langle v_{j}\right\rangle$ and $\left\langle v_{i}\right\rangle$, an identification with (3) is possible, which yields $c_{0}=\left\langle v_{i}\right\rangle\left\langle v_{j}\right\rangle, \quad c_{1}^{\text {pre }}=-\left\langle v_{i}\right\rangle, \quad c_{1}^{\text {post }}=-\left\langle v_{j}\right\rangle$, and $c_{2}^{\text {corr }}=1$.

Instead of an expansion in the postsynaptic rate $v_{i}$, an expansion in the membrane potential $u_{i}$ would also be possible. As mentioned in Sect. 2.1.1, $u_{i}$ and $v_{i}$ are related by (1). Hence, we can define a new function $\tilde{F}$ as

$\tilde{F}\left(w_{i j} ; u_{i}, v_{j}\right)=F\left(w_{i j} ; g\left(u_{i}\right), v_{j}\right)$

with Taylor expansion

$\frac{\mathrm{d}}{\mathrm{d} t} w_{i j}=c_{0}+c_{1}^{\text {pre }} v_{j}+b_{1}^{\text {post }} u_{i}+b_{2}^{\text {corr }} u_{i} v_{j}+\ldots$.

Here we have assumed that the voltage scale is chosen so that the resting potential is at $u_{i}=0$. The idea of an expansion in the membrane potential will be used again in Sect. 3.

\section{Spike-based learning}

The approach taken in this section can be seen as a generalization of the Taylor expansion in the rate model of Sect. 2 to the case of spiking neurons. We recall that we started our formulation of rate-based Hebbian learning from a general formula

$\frac{\mathrm{d}}{\mathrm{d} t} w_{i j}=F\left(w_{i j} ; v_{i}, v_{j}\right)$

where weight changes are given as a function of the weight $w_{i j}$ as well as of the pre- and postsynaptic rates $v_{j}$ and $v_{i}$ (see Eq. 2). The essential assumption was that neuronal activity is characterized by firing rates that change slowly enough to be considered as stationary. Hebbian rules followed then from a Taylor expansion of (12). In the following, we keep the idea of an expansion, but drop the assumption of rate-coded neurons.

A simple model of a spiking neuron is the leaky integrate-and-fire neuron (Lapicque 1907; Stein 1965). In its simplest form an integrate-and-fire neuron $i$ consists of a resistor $R$ in parallel with a capacitor $C$ driven by an external current $I_{i}$. The voltage $u_{i}$ across the capacitor and resistor is interpreted as the membrane potential. The voltage scale is chosen so that $u_{i}=0$ is the resting potential. The temporal evolution of $u_{i}$ is

$\tau_{\mathrm{m}} \frac{\mathrm{d} u_{i}}{\mathrm{~d} t}=-u_{i}+R I_{i}(t)$

where $\tau_{\mathrm{m}}=R C$ is the membrane time constant of the neuron. Spikes are formal events. We say that neuron $i$ has fired a spike if $u_{i}$ reaches at a time $t=t_{i}^{f}$ a threshold $\vartheta$. The form of the action potential is not described explicitly, but to mark the spike we can add a $\delta$ function $\delta\left(t-t_{i}^{f}\right)$. Immediately after spike firing, the potential $u_{i}$ is simply reset to a value $u_{\text {reset }}<\vartheta$. Integration of (13) is then resumed with $u_{\text {reset }}$ as the initial condition.

In a network of neurons, the input $I_{i}$ to neuron $i$ is due to the spikes of presynaptic neurons $j$. In the simplest model of a synapse, each presynaptic spike arrival evokes a postsynaptic current with a standard time course $\alpha$. The total input to neuron $i$ is then

$I_{i}=\sum_{j, f} w_{i j} \alpha\left(t-t_{j}^{f}\right)$

where the sum runs over all firing times $t_{j}^{f}$ of all presynaptic neurons. The factor $w_{i j}$ is the synaptic efficacy of a connection from a presynaptic neuron $j$ to a postsynaptic neuron $i$.

Since (13) is a linear equation, the integration can be performed for each term in the sum of (14) separately. The total membrane potential is then the sum of all the postsynaptic potentials caused by presynaptic firing plus the refractory effect of a negative reset potential. Given the last firing time $\hat{t}_{i}$ of neuron $i$, the result of the integration is therefore of the form (for $t>\hat{t}_{i}$ )

$u_{i}(t)=\eta\left(t-\hat{t}_{i}\right)+\sum_{j, f} w_{i j} \epsilon\left(t-\hat{t}_{i}, t-t_{j}^{f}\right)$

where

$\eta(t-\hat{t})=\delta\left(t-\hat{t}_{i}\right)+u_{\mathrm{reset}} \exp \left[\frac{-\left(t-\hat{t}_{i}\right)}{\tau_{\mathrm{m}}}\right]$

and

$\epsilon\left(t-\hat{t}_{i}, s\right)=\int_{0}^{t-\hat{t}_{i}} \alpha\left(s-s^{\prime}\right) \exp \left(-s^{\prime} / \tau_{\mathrm{m}}\right) \mathrm{d} s^{\prime}$.

The firing time $\hat{t}_{i}$ is updated whenever $u_{i}$ reaches the threshold $\vartheta$ from below. Equation (15) is the equation of the spike response model (SRM; Gerstner and van Hemmen 1992a; Gerstner 1995a; Kistler et al. 1997), a generalization of the leaky integrate-and-fire neuron. In the general SRM, the function $\eta\left(t-\hat{t}_{i}\right)$ describes the form of the action potential and the hyperpolarizing spike afterpotential that induces neuronal refractoriness. Each term $\epsilon\left(t-\hat{t}_{i}, t-t_{j}^{f}\right)$ describes the time course of a postsynaptic potential caused by presynaptic spike arrival at time $t-t_{j}^{f}$. Because of refractoriness, the postsynaptic potential $\epsilon$ has, in the general SRM, a nontrivial dependence upon the last postsynaptic firing 
time. In the following we neglect the dependence of the postsynaptic potential upon $t-\hat{t}_{i}$ and write

$u_{i}(t)=\eta\left(t-\hat{t}_{i}\right)+h_{i}(t)$

where $h_{i}(t)=\sum_{j} w_{i j} \epsilon\left(t-t_{j}^{f}\right)$ is the total postsynaptic potential. Thus, the internal state of spiking neurons is characterized by the membrane potential $u$ which in turn depends on the last output spike and the total postsynaptic potential (see Fig. 2). Equation (16) defines the simple spiking model $\mathrm{SRM}_{0}$.

We now return to the problem of Hebbian synaptic plasticity. As before, we start our formulation of Hebbian learning with the locality requirement. While the neuronal state of rate neurons was characterized by their firing rate $v$, the internal state of spiking neurons is characterized by their membrane potential $u$. The generalization of (12) to the case of spiking neurons is therefore

$$
\frac{\mathrm{d}}{\mathrm{d} t} w_{i j}(t)=F\left[w_{i j}(t) ; u_{i}^{\text {post }}\left(t^{\prime}\right), u_{j}^{\text {pre }}\left(t^{\prime \prime}\right)\right]
$$

where $F$ is now a functional of the time course of the preand postsynaptic membrane potentials at the location of the synapse. Our notation with $t^{\prime}$ and $t^{\prime \prime}$ is intended to indicate that the weight changes do not only depend on the momentary value of the pre- and postsynaptic potentials (at time $t$ ), but also on their history for $t^{\prime}<t$ and $t^{\prime \prime}<t$. The weight value $w_{i j}$ and the local values of pre- and postsynaptic membrane potentials are the essential variables that are available at the site of the synapse to control the up- and downregulation of synaptic weights. In detailed neuron models, $F$ would depend not only on the weight $w_{i j}$ and membrane potentials, but also on all other variables that are locally available at the site of the synapse. In particular, there could be a dependence upon the local calcium concentration. In the following we adopt the point of view that the calcium concentration is largely determined by the previous firing history, so that there is no need to introduce an additional explicit variable for calcium.

In analogy to the approach taken in Sect. 2, we now expand the right-hand side of (17) about the resting state $u_{i}^{\text {post }}=u_{j}^{\text {pre }}=u_{\text {rest }}$. For the sake of simplicity we shift the

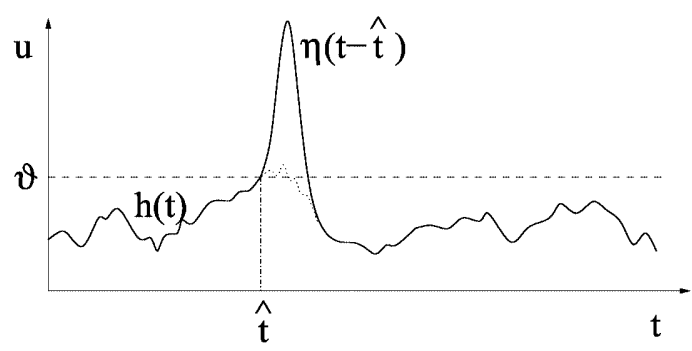

Fig. 2. The membrane potential of a spiking neuron $u(t)=h(t)+\eta(t-\hat{t})$ can be seen as a superposition of the total postsynaptic potential $h(t)$ generated by presynaptic spike arrival and the action potentials of the postsynaptic neuron. The time course of the action potential is $\eta(t-\hat{t})$, where $\hat{t}$ is the firing time of the last output spike. A backpropagating action potential could convey information about postsynaptic spike firing to the site of the synapse voltage scale so that $u_{\text {rest }}=0$. A Volterra expansion of (17) yields

$$
\begin{aligned}
\frac{\mathrm{d} w_{i j}}{\mathrm{dt}}= & c_{0}\left(w_{i j}\right)+\int_{0}^{\infty} \alpha_{1}^{\mathrm{pre}}\left(w_{i j} ; s\right) u_{j}^{\mathrm{pre}}(t-s) \mathrm{d} s \\
& +\int_{0}^{\infty} \alpha_{1}^{\mathrm{post}}\left(w_{i j} ; s^{\prime}\right) u_{i}^{\mathrm{post}}\left(t-s^{\prime}\right) \mathrm{d} s^{\prime} \\
& +\int_{0}^{\infty} \int_{0}^{\infty} \alpha_{2}^{\text {corr }}\left(w_{i j} ; s, s^{\prime}\right) u_{j}^{\mathrm{pre}}(t-s) u_{i}^{\text {post }}\left(t-s^{\prime}\right) \mathrm{d} s^{\prime} \mathrm{d} s \\
& +\ldots
\end{aligned}
$$

The next terms would be quadratic in $u_{i}^{\text {post }}$ or $u_{j}^{\text {pre }}$, and have been neglected. Equation (18) is the central result of this section. It provides a framework for the formulation of spike-based learning rules and may be seen as the generalization of the general rate-based model that we have derived in Sect. 2. The considerations summarized in the points discussed in Sects. 2.1.3-2.1.6 apply also to the case of spike-based learning rules, and will not be repeated here.

In order to establish a connection with various other formulations of spike-based learning rules, we consider the time course of the pre- and postsynaptic membrane potentials in more detail. At the presynaptic terminal, the membrane potential is most of the time at rest, except when an action potential arrives. Since the duration of each action potential is short, the presynaptic membrane potential can be approximated by a train of $\delta$ functions:

$u_{j}^{\mathrm{pre}}(t)=\sum_{f} \delta\left(t-t_{j}^{f}\right)$

where $t_{j}^{f}$ denotes the spike arrival times at the presynaptic terminal.

The situation at the postsynaptic site is somewhat more complicated. For the simple spike response model $\mathrm{SRM}_{0}$, the membrane potential can be written as

$u_{i}^{\text {post }}(t)=\eta\left(t-\hat{t}_{i}\right)+h_{i}(t)$

where $\hat{t}_{i}$ is the last postsynaptic firing time. In contrast to the usual interpretation of terms on the right-hand side of (20), the function $\eta$ is now taken as the time course of the backpropagating action potential (BPAP) at the location of the synapse. Similarly, $h_{i}(t)$ is the local postsynaptic potential at the synapse.

For a further simplification of (18), we need to make some approximations. Specifically we will explore two different approximation schemes. In the first scheme, we suppose that the dominating term on the right-hand side of (20) is the BPAP, while in the second scheme we neglect $\eta$ and consider $h$ as the dominant term. Let us discuss both approximations in turn.

\subsection{Sharply peaked backpropagating action potential}

We assume that the BPAP is sharply peaked, i.e., it has a large amplitude and short duration. In this case, the membrane potential of the postsynaptic neuron is 
dominated by the BPAP, and the term $h(t)$ in (20) can be neglected. Furthermore $\eta$ can be approximated by a $\delta$ function. The membrane potential at the postsynaptic site reduces then to a train of pulses

$u_{i}^{\text {post }}(t)=\sum_{f} \delta\left(t-t_{i}^{f}\right)$

where $t_{i}^{f}$ denotes the postsynaptic firing times. Equation (21) is a sensible approximation for synapses that are located on or close to the soma, for which the full somatic action potential is 'felt' by the postsynaptic neuron. For neurons with active processes in the dendrite that keep the BPAP well focused, (21) is also a reasonable approximation for synapses that are further away from the soma. A transmission delay for backpropagation of the spike from the soma to the site of the synapse can be incorporated at no extra cost.

If we insert (19) and (21) into (18), we obtain

$$
\begin{aligned}
\frac{\mathrm{d} w_{i j}}{\mathrm{dt}}= & c_{0}\left(w_{i j}\right)+\sum_{t_{j}^{f}} \alpha_{1}^{\mathrm{pre}}\left(w_{i j} ; t-t_{j}^{f}\right) \\
& +\sum_{t_{i}^{f}} \alpha_{1}^{\text {post }}\left(w_{i j} ; t-t_{i}^{f}\right) \\
& +\sum_{t_{j}^{f}} \sum_{t_{i}^{f}} \alpha_{2}^{\text {corr }}\left(w_{i j} ; t-t_{i}^{f}, t-t_{j}^{f}\right)+\ldots .
\end{aligned}
$$

We emphasize that weight changes are continuous. An isolated presynaptic spike at time $t_{j}^{f}$, for example, will cause a weight change that builds up during some time after $t_{j}^{f}$; the time course of the weight change $\mathrm{d} w_{i j} / \mathrm{d} t$ is described by $\alpha_{1}^{\mathrm{pre}}\left(w_{i j} ; t-t_{j}^{f}\right)$. The total weight change that accumulates for $t \rightarrow \infty$ is therefore $\Delta w_{i j}=\int_{0}^{\infty} \alpha_{1}^{\mathrm{pre}}\left(w_{i j} ; s\right) \mathrm{d} s$. Similarly, a single postsynaptic spike causes a weight change $\mathrm{d} w_{i j} / \mathrm{d} t$ with time course $\alpha_{1}^{\text {post }}\left(w_{i j} ; t-t_{i}^{f}\right)$. Finally, a combination of pre- and postsynaptic spikes within a short interval induces an additional weight change $\alpha_{2}^{\text {corr }}\left(w_{i j} ; t-t_{i}^{f}, t-t_{j}^{f}\right)$.

In typical plasticity experiments, the synaptic weight is monitored every few hundred milliseconds, so that the exact time courses of the functions $\alpha_{1}^{\text {pre }}, \alpha_{1}^{\text {post }}$, and $\alpha_{2}^{\text {corr }}$ are not measured. We now assume that the weight changes are rapid compared to the time scale of weight monitoring. In other words, we make the replacements

$\alpha_{1}^{\mathrm{pre}}\left(w_{i j} ; t-t_{j}^{f}\right) \longrightarrow c_{1}^{\mathrm{pre}}\left(w_{i j}\right) \delta\left(t-t_{j}^{f}\right)$

$\alpha_{1}^{\text {post }}\left(w_{i j} ; t-t_{i}^{f}\right) \longrightarrow c_{1}^{\text {post }}\left(w_{i j}\right) \delta\left(t-t_{i}^{f}\right)$

where

$$
\begin{gathered}
c_{1}^{\mathrm{pre}}\left(w_{i j}\right)=\int_{0}^{\infty} \alpha_{1}^{\mathrm{pre}}\left(w_{i j} ; s\right) \mathrm{d} s \\
c_{1}^{\text {post }}\left(w_{i j}\right)=\int_{0}^{\infty} \alpha_{1}^{\text {post }}\left(w_{i j} ; s\right) \mathrm{d} s
\end{gathered}
$$

are the accumulated weight changes. Note that we have chosen the same symbols $c_{1}^{\text {pre }}$ and $c_{1}^{\text {post }}$ as in (3). The reason is that, if pre- and postsynaptic spike trains are generated by Poisson processes with rates $v_{j}$ and $v_{i}$, respectively, then the expected weight change, (calculated from Eq. 18 without the correlation term or higherorder terms) is

$$
\left\langle\mathrm{d} w_{i j} / \mathrm{d} t\right\rangle=c_{0}\left(w_{i j}\right)+c_{1}^{\text {pre }}\left(w_{i j}\right) v_{j}+c_{1}^{\text {post }}\left(w_{i j}\right) v_{i}+\ldots
$$

which is identical to the corresponding terms in (3).

For the correlation term we exploit the invariance with respect to time translation, i.e., the final result should only depend on the time difference $t_{j}^{f}-t_{i}^{f}$. The weight update occurs at the moment of the postsynaptic spike if $t_{j}^{f}<t_{i}^{f}$, and at the moment of the presynaptic spike if $t_{j}^{f}>t_{i}^{f}$. Hence, the assumption of instantaneous update yields two terms:

$$
\begin{gathered}
\alpha_{2}^{\text {corr }}\left(w_{i j} ; t-t_{i}^{f}, t-t_{j}^{f}\right) \longrightarrow \\
\begin{cases}W\left(w_{i j} ; t_{j}^{f}-t_{i}^{f}\right) \delta\left(t-t_{j}^{f}\right) & \text { for } t_{i}^{f}<t_{j}^{f} \\
W\left(w_{i j} ; t_{j}^{f}-t_{i}^{f}\right) \delta\left(t-t_{i}^{f}\right) & \text { for } t_{i}^{f} \geq t_{j}^{f} .\end{cases}
\end{gathered}
$$

Thus, for sharply peaked BPAPs and rapid weight changes, we arrive at the notion of a time window $W$ for Hebbian synaptic plasticity (see Fig. 3). Such a 'learning window' has been used in numerous models of spike-time-dependent plasticity (Gerstner et al. 1993, 1996; Kempter et al. 1999; Roberts 1999; Kistler and van Hemmen 2000; Roberts and Bell 2000; van Rossum et al. 2000; Song et al. 2000; Rubin et al. 2001) and also been measured in experimental preparations (Bell et al. 1997; Magee and Johnston 1997; Markram et al. 1997; Bi and Poo 1998, 1999; Debanne et al. 1998; Zhang et al. 1998). Time windows have also been used in rate-based models with time-dependent firing rates (Herz et al. 1989; Minai and Levy 1993; Abbott and Blum 1996; Gerstner and Abbott 1997). The relevance of the learning window $W\left(t_{j}^{f}-t_{i}^{f}\right)$ is discussed in Sects. 4 and 5.

To explore the relation between the learning window and the correlation term in the rate equation (3), we assume that the firing times of the pre- and the postsynaptic neuron are generated by independent Poisson process with constant rates $v_{j}$ and $v_{i}$, respectively. The expected weight evolution is then

$$
\begin{aligned}
\left\langle\frac{\mathrm{d}}{\mathrm{d} t} w_{i j}\right\rangle= & c_{0}\left(w_{i j}\right)+c_{1}^{\mathrm{pre}}\left(w_{i j}\right) v_{j}+c_{1}^{\text {post }}\left(w_{i j}\right) v_{i} \\
& +\left[\int_{0}^{\infty} W(s) \mathrm{d} s\right] v_{i} v_{j} .
\end{aligned}
$$

Thus, the integral over the learning window plays the role of the correlation parameter $c_{2}^{\text {corr }}$ in (3).

\subsection{No backpropagating action potential}

In the second approximation scheme, we assume that the membrane potential at the location of the synapse is dominated by the slowly varying potential $h_{i}(t)$. This is, for example, a valid assumption in voltage-clamp experiments where the postsynaptic neuron is artificially kept at a constant membrane potential $h^{\text {post }}$. This is also a good approximation for synapses that a located far away from the soma on a passive dendrite, so that the 
A

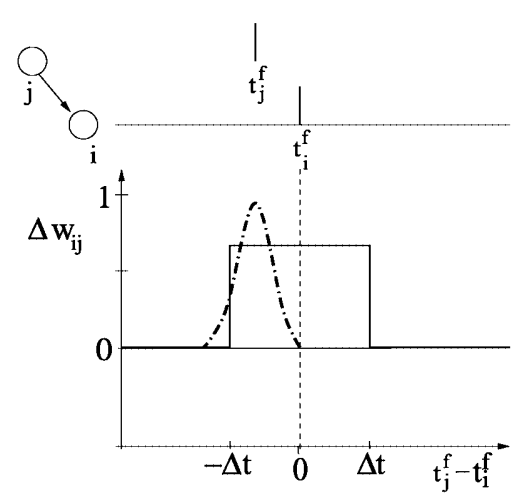

B

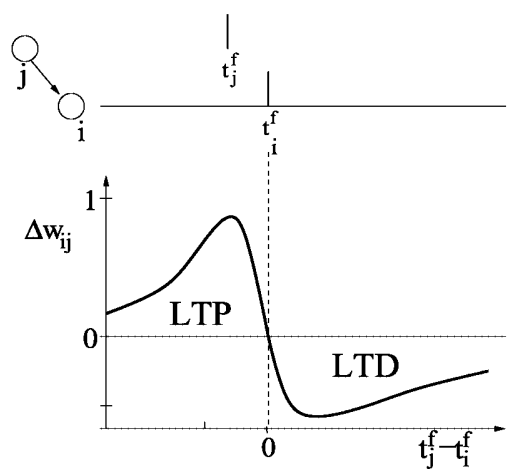

C

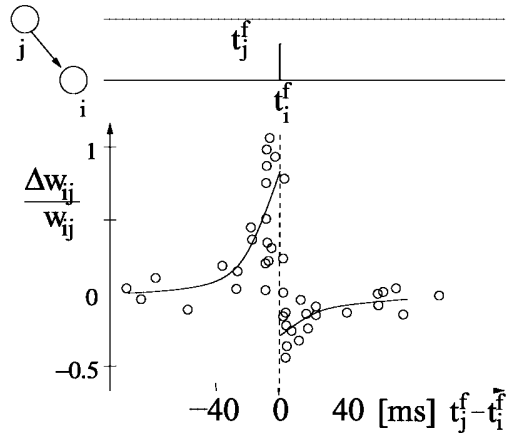

Fig. 3A-C. Learning window. The change $\Delta w_{i j}$ of the synaptic efficacy depends on the timing of pre- and postsynaptic spikes. A The solid line indicates a rectangular time window as it is often used in standard Hebbian learning. The synapse is increased if the pre- and the postsynaptic neuron fire simultaneously with a temporal resolution $\Delta t$. The dashed-dotted line shows an asymmetric learning window useful for sequence learning (Herz et al. 1989; Gerstner and van Hemmen 1993). The synapse is strengthened if the presynaptic spike arrives slightly before the postsynaptic one, and is therefore

backpropagation of somatic action potentials is negligible.

Let us consider a voltage-clamp experiment where $h_{i}(t)$ is kept at a constant level $h^{\text {post }}$. As before, we suppose that weight changes are rapid. If we insert $u_{j}^{\text {pre }}(t)=\sum_{f} \delta\left(t-t_{j}^{f}\right)$ and $u_{i}^{\text {post }}(t)=h^{\text {post }}$ into (18), we obtain

$$
\begin{aligned}
\frac{\mathrm{d} w_{i j}}{\mathrm{~d} t}= & c_{0}\left(w_{i j}\right)+\sum_{f} c_{1}^{\mathrm{pre}}\left(w_{i j}\right) \delta\left(t-t_{j}^{f}\right) \\
& +b_{1}^{\text {post }}\left(w_{i j}\right) h^{\text {post }} \\
& +b_{2}^{\text {corr }}\left(w_{i j}\right) h^{\text {post }} \sum_{f} \delta\left(t-t_{j}^{f}\right)+\ldots
\end{aligned}
$$

where

$$
\begin{aligned}
c_{1}^{\text {pre }}\left(w_{i j}\right) & =\int_{0}^{\infty} \alpha_{1}^{\text {pre }}\left(w_{i j} ; s\right) \mathrm{d} s \\
b_{1}^{\text {post }}\left(w_{i j}\right) & =\int_{0}^{\infty} \alpha_{1}^{\text {post }}\left(w_{i j} ; s\right) \mathrm{d} s \\
b_{2}^{\text {corr }}\left(w_{i j}\right) & =\int_{0}^{\infty} \int_{0}^{\infty} \alpha_{2}^{\text {corr }}\left(w_{i j} ; s, s^{\prime}\right) \mathrm{d} s \mathrm{~d} s^{\prime} .
\end{aligned}
$$

Equation (27) is the starting point of the theory of spikebased learning of (Fusi et al. 2000). Weight changes are triggered by presynaptic spikes, and the direction and value of the weight update depends on the postsynaptic membrane potential. We note the close relation to (11). In our framework, (27) is a special case of the slightly more general (18).

\section{Analysis of Hebbian learning}

It is well known that rate-based learning rules are sensitive to the spatial correlations in the input (Hertz partially 'causal' in firing it. B An asymmetric biphasic learning window as introduced in model studies of delay selection (Gerstner et al. 1996). A synapse is strengthened (long-term potentiation, LTP) if the presynaptic spike arrives slightly before the postsynaptic one, but is decreased (long-term depression, LTD) if the timing is reversed. The biphasic learning window is sensitive to the temporal contrast in the input. C Experimental results have confirmed the existence of biphasic learning windows. Data points redrawn after the experiments of Bi and Poo (1998)

et al. 1991). If the postsynaptic neuron is modeled as a linear unit:

$v_{i}(t)=\sum_{k} w_{i k} v_{k}(t)$

then the standard Hebbian learning rule $\mathrm{d} w_{i j} / \mathrm{d} t$ $=c_{2}^{\text {corr }} v_{i} v_{j}$ yields an expected weight evolution

$\left\langle\frac{\mathrm{d}}{\mathrm{d} t} w_{i j}\right\rangle=c_{2}^{\mathrm{corr}} \sum_{k} w_{i k}\left\langle v_{k}(t) v_{j}(t)\right\rangle$

which is in the direction of the principal eigenvector of the spatial correlation matrix

$C_{j k}=\left\langle v_{k}(t) v_{j}(t)\right\rangle$.

Angular brackets denote an expectation value. If learning is slow, ${ }^{1}$ then the actual weight vector in fact stays close to the expected one so that the angular brackets on the left-hand side of (29) can be dropped.

We may introduce a vector $\boldsymbol{w}_{i}$ for the set of synapses that converge on the postsynaptic neuron $i$. The correlation between presynaptic neurons can be denoted by a matrix $\mathbf{C}$ with components $C_{j k}$. In matrix notation, (29) is

$\frac{\mathrm{d}}{\mathrm{d} t} \boldsymbol{w}_{i}=c_{2}^{\operatorname{corr}} \mathbf{C} \boldsymbol{w}_{i}$

Since the correlation matrix is positive definite, the weight vector grows exponentially for in standard Hebbian learning $\left(c_{2}^{\text {corr }}>0\right)$.

\footnotetext{
${ }^{1}$ The weight vector should change only by a small amount during the time needed to obtain a representative sample of the input statistics. This can always be achieved by taking $c_{2}^{\text {corr }} \ll 1$. We say in this case that the time scale of learning and that of the input are separated.
} 
To avoid unlimited growth of weights and to introduce competivity between the synapses converging onto the same postsynaptic neuron, (29) is usually combined with a suitable normalization procedure (Oja 1982; Miller and MacKay 1994). As an example, we consider subtractive normalization (Miller and MacKay 1994). To see how this works, we assume that during a single trial of duration $\mathscr{T}$, the standard Hebbian rule $\mathrm{d} w_{i j} / \mathrm{d} t=c_{2}^{\text {corr }} v_{i} v_{j}$ would yield a 'raw' weight change $\Delta \tilde{w}_{i j}$. In order to guarantee that the sum of the weights $\sum_{k} w_{i k}$ does not change, all weights are reduced a posteriori by an amount $N^{-1} \sum_{k} \Delta \tilde{w}_{i k}$ where $N$ is the number of synapses converging onto the same postsynaptic neuron $i$. Overall the two steps (i.e., 'raw' change and subsequent reduction) amount to a new learning rule:

$\frac{\mathrm{d}}{\mathrm{d} t} w_{i j}=c_{2}^{\mathrm{corr}} v_{i} v_{j}-N^{-1} c_{2}^{\operatorname{corr}} v_{i} \sum_{k=1}^{N} v_{k}$.

The first term on the right-hand side of (32) is the standard learning rule, and the second term the subtractive normalization term. If we write the postsynaptic rate as $v_{i}=\sum_{k} w_{i k} v_{k}$ and take as before the ensemble average, we find

$\frac{\mathrm{d}}{\mathrm{d} t} \boldsymbol{w}=c_{2}^{\mathrm{corr}}(\mathbf{C}-\overline{\mathbf{C}}) \boldsymbol{w}$

where $\overline{\mathbf{C}}$ is a matrix with components $\bar{C}_{k j}=\sum_{n} C_{n j}$. Equation (33) is the analog of (31) for the case of subtractive weight normalization. It is usually combined with hard bounds $0 \leq w_{i j}<w^{\max }$.

Note that the rule (32) is nonlocal, since the weight change at the synapse from $j$ to $i$ depends on the firing rate of other neurons $k$. We show in Sect. 2 that implicit subtractive normalization can also be achieved by local rules via a stabilization of postsynaptic firing rates.

\subsection{Spike-based learning}

An analogous argument can be developed for spikebased learning. The linear rate model in (28) is replaced by a stochastically spiking neuron model with instantaneous firing rate:

$$
\begin{aligned}
v_{i}(t) & =\sum_{k} w_{i k} \sum_{f} \epsilon\left(t-t_{k}^{f}\right) \\
& =\sum_{k} w_{i k} \int_{0}^{\infty} \epsilon(s) S_{k}(t-s) \mathrm{d} s
\end{aligned}
$$

where $t_{k}^{f}$ denotes the time of presynaptic spike arrival at the synapse from neuron $k$ to neuron $i . S_{k}(t)$ $=\sum_{f} \delta\left(t-t_{k}^{f}\right)$ is the presynaptic spike train. The time course of the postsynaptic potential is described by the function $\epsilon$, which is normalized to $\int_{0}^{\infty} \epsilon(s) \mathrm{d} s=1$. We implicitly assume that the right-hand side of (34) is positive (which is guaranteed if $w_{i k} \geq 0$ and $\epsilon \geq 0$ ). The left-hand side can then be taken as the instantaneous rate (or stochastic intensity) of an inhomogeneous Poisson process. We call (34) a linear Poisson neuron.
We assume that input spike trains have stationary statistics with known mean and correlations. Specifically, input spikes at a given synapse $j$ are generated by a doubly stochastic point process. Spikes are generated by an inhomogeneous Poisson process with instantaneous rate $v_{j}(t)$. The rate itself is drawn from a distribution with constant expectation value $\left\langle v_{j}(t)\right\rangle=v^{\text {pre }}$ and correlations $\left\langle v_{j}(t) v_{k}\left(t^{\prime}\right)\right\rangle=C_{j k}\left(t-t^{\prime}\right)$. We suppose that all presynaptic spike trains have identical properties. In particular, we require that $\left\langle v_{j}(t)\right\rangle=v^{\text {pre }}$ independent of $j$, and $N^{-1} \sum_{k=1}^{N} C_{i k}(s)=\bar{C}(s)$ independent of $i$. Since the expected input rates are constant, the expected output rate $v^{\text {post }} \equiv\left\langle v_{i}(t)\right\rangle=v^{\text {pre }} \sum_{j} w_{i j}(t)$ is a slow function of time which changes only as the synaptic efficacies adapt.

In order to discuss the weight dynamics we start from the general spike-based learning equation that has been developed in Sect. 3 for the case of sharply peaked BPAPs (see Eq. 22). Throughout the following we assume that weight changes $\Delta w_{i j} /\left|w_{i j}\right|$ are small during the time that is necessary to approximately sample the input statistics. In this case we can separate the time scale of learning from that of the neuronal dynamics. The righthand side of (22) is then 'self-averaging' (Kempter et al. 1999), so that the evolution of the weight vector (22) is given by (Kempter et al. 1999; Kistler and van Hemmen 2000; Roberts 2000c)

$$
\begin{aligned}
\frac{\mathrm{d}}{\mathrm{d} t} w_{i j}(t)= & c_{0}+c_{1}^{\mathrm{pre}}\left\langle\left\langle S_{j}(t)\right\rangle\right\rangle+c_{1}^{\mathrm{post}}\left\langle\left\langle S_{i}(t)\right\rangle\right\rangle \\
& +\int_{-\infty}^{\infty} W(s)\left\langle\left\langle S_{j}(t) S_{i}(t-s)\right\rangle\right\rangle \mathrm{d} s .
\end{aligned}
$$

Here we have introduced

$$
\begin{aligned}
& c_{1}^{\text {pre }}\left(w_{i j}\right)=\int_{0}^{\infty} \alpha_{1}^{\text {pre }}\left(w_{i j} ; s\right) \mathrm{d} s \\
& c_{1}^{\text {post }}\left(w_{i j}\right)=\int_{0}^{\infty} \alpha_{1}^{\text {post }}\left(w_{i j} ; s\right) \mathrm{d} s
\end{aligned}
$$

and

$$
W\left(w_{i j} ; t_{j}^{f}-t_{i}^{f}\right)=\int_{\max \left(t_{i}^{f}, t_{j}^{f}\right)}^{\infty} \alpha_{2}^{\mathrm{corr}}\left(w_{i j} ; t^{\prime}-t_{i}^{f}, t^{\prime}-t_{j}^{f}\right) \mathrm{d} t^{\prime}
$$

The double angular brackets in (35) denote the average over the input statistics. The notation with double angular brackets emphasizes that the underlying process is a doubly stochastic one: we first have to calculated the expected number of spikes, given the rates; and then we have to average over the rates. The double angular brackets on the left-hand side have been dropped, because of the self-averaging property.

For an interpretation of (35) we start with the terms that are linear in the spike trains. We first calculate the expected number of spikes given the rates and take then the expectation of the rates:

$$
\left\langle\left\langle S_{j}(t)\right\rangle\right\rangle=\left\langle v_{j}(t)\right\rangle=v^{\mathrm{pre}}
$$




$$
\begin{aligned}
\left\langle\left\langle S_{i}(t)\right\rangle\right\rangle=\left\langle v_{i}(t)\right\rangle & =\sum_{j} w_{i j} \int_{0}^{\infty} \epsilon(s)\left\langle\left\langle S_{j}(t-s)\right\rangle\right\rangle \mathrm{d} s \\
& =\sum_{j} w_{i j}(t) v^{\text {pre }}=v^{\text {post }}(t)
\end{aligned}
$$

Finally, the term $\left\langle S_{j} S_{i}\right\rangle$ on the right-hand side of (35) describes the correlation between input and output at the level of spikes. We may summarize (35) by saying that the evolution of the weight vector is driven by the expected firing rates and by correlations on the time scale of the learning window.

The correlation term depends not only on the input statistics, but also on the dynamics of the neuron model under consideration. Since we have assumed that learning is a slow process, the correlation term can be evaluated for constant weights $w_{i j}, 1 \leq i \leq N$. For the linear Poisson neuron model, the correlations between pre- and postsynaptic spike trains can be written as (Kempter et al. 1999)

$$
\left\langle\left\langle S_{j}(t) S_{i}(t-s)\right\rangle\right\rangle=\left\langle v_{i}(t-s) v_{j}(t)\right\rangle+\left\langle v_{j}\right\rangle w_{i j}(t) \epsilon(-s)
$$

with a postsynaptic firing rate $v_{i}(t)$ given by (34).

Substituting (37)-(39) into (35) we obtain

$$
\begin{aligned}
\frac{\mathrm{d}}{\mathrm{d} t} w_{i j}(t)= & c_{0}+c_{1}^{\mathrm{pre}} v^{\mathrm{pre}}+c_{1}^{\text {post }} v^{\text {post }}(t) \\
& +\left[\int_{-\infty}^{\infty} W(s) \mathrm{d} s\right] v^{\text {post }}(t) v^{\text {pre }} \\
& +\sum_{k=1}^{N} w_{i k}(t)\left[Q_{j k}+\delta_{j k} v^{\text {pre }} \int_{-\infty}^{0} W(s) \epsilon(-s) \mathrm{d} s\right]
\end{aligned}
$$

where

$$
Q_{j k}=\int_{-\infty}^{\infty} \mathrm{d} s W(s) \int_{0}^{\infty} \mathrm{d} s^{\prime} \epsilon\left(s^{\prime}\right) C_{j k}^{0}\left(s+s^{\prime}\right) .
$$

The important factor in (41) is the spatiotemporal input covariance function

$C_{j k}^{0}(s)=\left\langle\left[v_{j}(t)-v^{\mathrm{pre}}\right]\left[v_{k}(t-s)-v^{\mathrm{pre}}\right]\right\rangle$

which is convolved with the learning window $W$ and the postsynaptic potential $\epsilon$. Thus the correlations between pre- and postsynaptic neuron in (35) has been transformed into spatiotemporal correlations in the input.

To summarize this section, we have solved the dynamics of spike-time-dependent plasticity under the assumption that learning is slow compared to the variations in the input. For the linear Poisson neuron, i.e., a stochastically spiking neuron model, the spike-tospike correlations between pre- and postsynaptic firings can be evaluated. The final result is a learning equation where weight changes are driven by the expected input rates as well as the spatial and temporal correlations of the input.

\subsection{Implicit subtractive weight normalization}

In this section we want to show that, for a suitable choice of parameters, synaptic plasticity leads to an intrinsic stabilization of the postsynaptic firing rate and, hence, to a normalization of weights that is akin to subtractive normalization (Kempter et al. 1999, 2001; Song et al. 2000). To do so we proceed in two steps (in Sects. 4.2.1 and 4.2.2). As a 'warm up' we focus in a first step on Poisson spike trains without correlations and show that for a suitable choice of parameters the rate of the postsynaptic neuron is stabilized. In a second step, we extend this result to the more interesting case including correlations in the input and show that rate stabilization implies subtractive weight normalization.

4.2.1 No correlations. First, we show that for a suitable choice of parameters, the output rate approaches a stable fixed point. To do so, we consider a linear Poisson neuron that receives input from $N$ presynaptic neurons with spike activity described by independent Poisson processes with rate $v^{\text {pre }}$. The postsynaptic neuron is thus firing at a rate $v^{\text {post }}(t)=v^{\text {pre }} \sum_{j=1}^{N} w_{i j}(t)$. Since the input is described as independent Poisson processes, the correlations $Q_{j k}$ in (40) vanish, so we obtain

$$
\begin{aligned}
\frac{\mathrm{d}}{\mathrm{d} t} w_{i j}(t)= & c_{0}+c_{1}^{\mathrm{pre}} v^{\mathrm{pre}}+c_{1}^{\mathrm{post}} v^{\mathrm{post}}(t) \\
& +\bar{W} v^{\mathrm{pre}} v^{\mathrm{post}}(t) \\
& +w_{i j}(t) v^{\mathrm{pre}} W_{-}
\end{aligned}
$$

where $\bar{W}=\int_{-\infty}^{\infty} W(s) \mathrm{d} s$ and $W_{-}=\int_{0}^{\infty} x \epsilon(s) W(-s) \mathrm{d} s$. In this particularly simple case the weight dynamics is characterized by a fixed point for the postsynaptic firing rate $v^{\text {post }}(t)=v_{\mathrm{FP}}$. To see this we multiply (43) by $v^{\text {pre }}$ and sum over $j$. The left-hand side becomes $\mathrm{d} v^{\text {post }} / \mathrm{d} t$, which we set equal to zero to find the fixed point:

$v_{\mathrm{FP}}=-\frac{c_{0}+c_{1}^{\mathrm{pre}} p^{\text {pre }}}{c_{1}^{\text {post }}+v^{\mathrm{pre}} \bar{W}+N^{-1} W_{-}}$.

This fixed point is attractive if the denominator is negative. Since $v^{\text {post }}$ is a firing rate, we have the additional requirement that $v_{\mathrm{FP}} \geq 0$. Altogether we thus have two conditions for the parameters of the learning rule: (i) $c_{1}^{\text {post }}+v^{\text {pre }} \bar{W}+N^{-1} W_{-}<0$, and (ii) $c_{0}+c_{1}^{\text {pre }} v^{\text {pre }} \geq 0$. Note that we would obtain - apart from the term $\left(N^{-1} W_{-}\right)$- a completely analogous result from the rate formulation in (3) if we identify $c_{2}^{\text {corr }}=\bar{W}$. Furthermore, note that the linearity is not essential for the stabilization of the postsynaptic rate. Any model where the output rate is a monotonous function of the sum of the synaptic weights yields qualitatively the same result.

4.2.2 Input correlations. We now extend the above results and show that output rate stabilization implies weight normalization even in the presence of input 
correlations. As before we introduce the notations $\bar{W}=\int_{-\infty}^{\infty} W(s) \mathrm{d} s$ and $W_{-}=\int_{-\infty}^{0} W(s) \epsilon(-s) \mathrm{d} s$. We expand and reorder the terms on the right-hand side of (40) into the following form:

$$
\begin{aligned}
\frac{\mathrm{d}}{\mathrm{d} t} w_{i j}(t)= & \left\{c_{0}+c_{1}^{\mathrm{pre}} v^{\mathrm{pre}}+c_{1}^{\mathrm{post}} v^{\mathrm{post}}(t)\right. \\
& +\bar{W} v^{\mathrm{pre}} v^{\mathrm{post}}(t)+W_{-} N^{-1} v^{\mathrm{post}}(t) \\
& \left.+\bar{Q} \frac{v^{\mathrm{post}}(t)}{v^{\mathrm{pre}}}\right\} \\
& +W_{-}\left[w_{i j}(t) v^{\mathrm{pre}}-N^{-1} v^{\mathrm{post}}(t)\right] \\
& +\sum_{k} w_{i k}\left[Q_{k j}-\bar{Q}\right] .
\end{aligned}
$$

Here $\bar{Q}=N^{-1} \sum_{k} Q_{i k}$ with $Q_{i k}$ given by (41). For a discussion of (45), let us first consider the expected postsynaptic rate $v^{\text {post }}(t)=\sum_{j} w_{i j}(t) v^{\text {pre }}$. Its temporal derivative can be found by taking the sum over $j$ on the right-hand side of (45) and multiplying by $v^{\text {pre }}$. The terms outside the curly braces in (45) cancel each other after summation, and the terms inside the braces yield a linear equation

$\frac{\mathrm{d} v^{\text {post }}(t)}{\mathrm{d} t}=\gamma\left(v^{\text {post }}-v_{\mathrm{FP}}\right)$

with a fixed point

$v_{\mathrm{FP}}=-\frac{c_{0}+c_{1}^{\text {pre }} v^{\text {pre }}}{c_{1}^{\text {post }}+v^{\text {pre }} \bar{W}+N^{-1} W_{-}+\bar{Q}\left(v^{\text {pre }}\right)^{-1}}$.

The fixed point of the mean postsynaptic rate is found to be stable if

$\gamma=c_{1}^{\text {post }}+v^{\text {pre }} \bar{W}+N^{-1} W_{-}+\bar{Q}\left(v^{\text {pre }}\right)^{-1}<0$.

To proceed with the analysis let us suppose that $\gamma \ll 0$. This can always be achieved if either $c_{1}^{\text {post }}$ or $\bar{W}$ is sufficiently negative. In this case, the effective time constant $\tau_{\text {eff }}=-1 / \gamma$ is short, so that the fixed point is attained rapidly. After convergence towards the fixed point, the summed weights $\sum_{j} w_{i j}$ remain constant and the terms within the curly brackets in (45) cancel each other. The remaining terms on the right-hand side of (45) determine the evolution of the weight vector. If we switch to matrix notation we have

$\frac{\mathrm{d}}{\mathrm{d} t} \boldsymbol{w}=[\mathbf{Q}-\overline{\mathbf{Q}}] \boldsymbol{w}+W_{-}\left[v^{\mathrm{pre}} \boldsymbol{w}-N^{-1} v_{\mathrm{FP}} \boldsymbol{n}\right]$

where $\boldsymbol{n}=(1,1, \ldots, 1)^{\mathrm{T}}$ and $\overline{\mathbf{Q}}$ denotes the matrix where all elements have the same value $\bar{Q}$. The result is similar to subtractive weight normalization (see Eq. 33) with a few specific differences - the spatial correlation matrix $\mathbf{C}$ that appears in rate-formulations of Hebbian learning (Miller and MacKay 1994), has been replaced by the matrix $\mathbf{Q}$ that describes the spatiotemporal covariance on the time scale of the learning window and postsynaptic potential. Thus, the learning rule behaves similar to Sejnowski's covariance rule (see Eq. 9). The additional spike-to-spike correlations that appear in spiking neuron models give rise to the second term on the right-hand side of (49). This tends to stabilize synapses that have large weights, and decrease synapses with small weights. If we neglect the spike-to-spike correlations, the dynamics of the weight vector is dominated by the eigenvector of the matrix $\mathbf{Q}-\overline{\mathbf{Q}}$ with the largest eigenvalue. Equation (49) can be seen as the generalization of the simple Hebbian learning rule (31) to the case of spike-based learning.

We recall that the coefficients $c_{0}, c_{1}^{\text {pre }}$, and $c_{1}^{\text {post }}$, and the learning window $W$ depend, in general, on the current weight value $w_{i j}$. In the above derivation we have assumed that these values are constant. It is, however, possible to set upper and lower bounds for the synaptic efficacies $w_{i j}$ (i.e., weight changes are zero) if $w_{i j}>w^{\max }$ or $w_{i j}<0$. It is straightforward to extend the above arguments to this case (Kempter et al. 1999). More realistically, we could also assume an explicit weight dependence where all positive terms have a saturating factor $\left(w_{i j}-w^{\max }\right)$ and all negative terms a decay factor $w_{i j}$. With these dependencies it is again possible to to analyze the weight dynamics (Kistler and van Hemmen 2000; van Rossum et al. 2000; Rubin et al. 2001; Kistler 2002).

\section{Discussion: learning windows}

The theoretical framework developed above shows that time windows of Hebbian synaptic plasticity arise naturally in spike-time-dependent plasticity. In this section, we place the learning window into the context of the experimental and modeling literature. For a modern review of experiments on Hebbian plasticity, see Bi and Poo (2001).

In early experimental paradigms neurons were stimulated by bursts of presynaptic spikes, and so the temporal resolution was not sufficient to measure the time course in detail. Nevertheless, even then it was clear that there were temporal contiguity requirements for Hebbian learning (Levy and Stewart 1983; Gustafsson et al. 1987; Debanne et al. 1994). Recent advances in electrophysiological methods allow intracellular recordings to be obtained from several neurons simultaneously, so that the timing windows of Hebbian synaptic plasticity have been measured with great detail (Bell et al. 1997; Markram et al. 1997; Magee and Johnston 1997; Bi and Poo 1998, 1999; Debanne et al. 1998; Zhang et al. 1998). One of the surprising findings has been that depending on the exact timing between pre- and postsynaptic spikes, synaptic weights are either increased or decreased (Fig. 3C). At excitatory synapses, a presynaptic spike that arrives slightly before the postsynaptic spike induces potentiation. This is in agreement with the 'causality' that is implicit in Hebb's formulation: if a presynaptic spike arrives slightly before a postsynaptic one, it is likely that this spike 'takes part in firing' the postsynaptic one. However, a presynaptic spike that arrives slightly after postsynaptic firing leads to synaptic depression. Thus the learning window $W\left(t_{i}^{f}-t_{j}^{f}\right)$ is not 
only asymmetric [which means that the maximum of the function $W(x)$ is not centered around $x=0$ ] but also biphasic (which means that it contains both excitation and depression).

A similar asymmetric biphasic learning window has, in fact, been introduced in the modeling literature (Gerstner et al. 1996), well before precise experimental results have been available (Fig. 3B). Even though biphasic learning windows with LTP and long-term depression (LTD) are not a strict prediction of the theory, they have several useful properties that make them attractive candidates for modeling. What are these properties?

It has been recognized early on that asymmetric learning windows where the maximum is shifted to a value $x<0$ (i.e., synapses are optimally strengthened if the presynaptic spike arrives slightly before the postsynaptic one) are ideally suited to sequence learning (Herz et al. 1989; Gerstner and van Hemmen 1993; Minai and Levy 1993; Abbott and Blum 1996). The reason is that such a shifted learning window implements the 'causality' condition (Fig. 3A). If, during learning, neuron $j$ was systematically firing a few milliseconds before neuron, the synapse from $j$ to $i$ is strengthened since $j$ 'takes part in firing' neuron $i$. After learning, the activity of neuron $j$ helps to induce activity of the postsynaptic neurons $i$, so that the sequence 'first $j$ then $i$ ' is recalled. In these models the width of the learning window (i.e., the range of $x$ during which the value $W(x)$ is significantly above zero) was on the same timescale as the sequence itself. In rate models where the activity evolves on a timescale of a few hundred milliseconds, the learning window was taken to have a width of a hundred milliseconds (Abbott and Blum 1996); in spiking models where the spatiotemporal sequence of spike patterns evolves on a time scale of 1 or $2 \mathrm{~ms}$, the width of the learning window was taken equal to $1 \mathrm{~ms}$ (Gerstner and van Hemmen 1993). If the width of the learning window were to be taken much broader, then the learning rule would strengthen not only the 'correct' synapses that help to recall the sequence, but also many other synapses such that the success of learning would be weakened or even wiped out.

A biphasic learning rule that contains both potentiation and depression introduces competition between the synapses (Gerstner et al. 1996, 1997; Kempter et al. 1999; Kistler and van Hemmen 2000; Song et al. 2000). For an appropriate choice of parameters, only those synapses that encode the optimal timing between preand postsynaptic neuron are strengthened, while a 'typical' synapse is depressed. As a result, the learning rule is sensitive to temporal structure in the input that can be fast compared to the width of the positive phase of the learning window (Gerstner et al. 1996). It is thus the causality condition (LTP for a timing 'first pre- then postsynaptic neuron') in combination with competition (induced by LTD for a sequence 'first post- then presynaptic neuron') that makes biphasic learning windows attractive candidates for learning.

During the last 5 years, asymmetric Hebbian learning rules with biphasic learning windows have therefore attracted a large interest in the modeling community
(Kempter et al. 1999; Roberts 1999, 2000; Kistler and van Hemmen 2000; van Rossum et al. 2000; Song et al. 2000; Xie and Seung 2000; Kempter et al. 2001; Rubin et al. 2001; Senn et al. 2002). Competition between synapses has been understood to arise from an intrinsic stabilization of postsynaptic firing rates (Gerstner et al. 1997b; Roberts 2000; van Rossum et al. 2000; Song et al. 2000; Xie and Seung 2000; Kempter et al. 2001). It has been shown that a biphasic learning window can be mapped to rate models where learning is driven by the derivative of the postsynaptic firing rate (Roberts 1999; Xie and Seung 2000). Spike-time-dependent plasticity is thought to play a major role in sensory image cancellation of electric fish (Roberts 2000) and in the tuning of delay lines in the barn owl auditory system (Gerstner et al. 1996). While most models of spike-time-dependent plasticity start with a given form of the learning window, the time course of the learning window itself can also be understood in terms of microscopic models (Gerstner et al. 1998; Senn et al. 2001; Shouval et al. 2002).

\section{References}

Abbott LF, Blum KI (1996) Functional significance of long-term potentiation for sequence learning and prediction. Cereb Cortex 6: 406-416

Bell C, Han V, Sugawara Y, Grant K (1997) Synaptic plasticity in a cerebellum-like structure depends on temporal order. Nature 387: 278-281

Bi G, Poo M (1998) Synaptic modifications in cultured hippocampal neurons: dependence on spike timing, synaptic strength, and postsynaptic cell type. J Neurosci 18: 10464 10472

Bi G, Poo M (1999) Distributed synaptic modification in neural networks induced by patterned stimulation. Nature 401: 792796

Bi G, Poo M (2001) Synaptic modification of correlated activity: Hebb's postulate revisited. Annu Rev Neurosci 24: 139-166

Bliss TVP, Collingridge GL (1993) A synaptic model of memory: long-term potentiation in the hippocampus. Nature 361: 31-39

Brown TH, Ganong AH, Kairiss EW, Keenan CL, Kelso SR (1989) Long-term potentation in two synaptic systems of the hippocampal brain slice. In: Byrne J, Berry W (eds) Neural models of plasticity. Academic, San Diego, pp 266-306

Brown TH, Zador AM, Mainen ZF, Claiborne BJ (1991) Hebbian modifications in hippocampal neurons. In: Baudry M, Davis J (eds) Long-term potentiation. MIT Press, Cambridge, Mass., pp 357-389

Carpenter G, Grossberg S (1987) Art 2: Self-organization of stable category recognition codes for analog input patterns. Appl Opt 26: 4919-4930

Debanne D, Gähwiler BH, Thompson SM (1994) Asynchronous pre- and postsynaptic activity induces associative long-term depression in area CA1 of the rat hippocampus in vitro. Proc Natl Acad Sci USA 91: 1148-1152

Debanne D, Gähwiler B, Thompson S (1998) Long-term synaptic plasticity between pairs of individual CA3 pyramidal cells in rat hippocampal slice cultures. J Physiol (Lond) 507: 237-247

Fusi S, Annunziato M, Badoni D, Salamon A, Amit DJ (2000) Spike-driven synaptic plasticity: theory, simulation, VLSI implementation. Neural Comput 12: 2227-2258

Gerstner W (1995) Time structure of the activity in neural network models. Phys Rev E 51: 738-758

Gerstner W, Abbott LF (1997) Learning navigational maps through potentiation and modulation of hippocampal place cells. J Comput Neurosci 4: 79-94 
Gerstner W, van Hemmen JL (1992) Associative memory in a network of 'spiking' neurons. Network 3: 139-164

Gerstner W, van Hemmen JL (1993) Coherence and incoherence in a globally coupled ensemble of pulse emitting units. Phys Rev Lett 71: 312-315

Gerstner W, Kistler WK (2002) Spiking neuron models. Cambridge University Press, Cambridge

Gerstner W, Ritz R, van Hemmen JL (1993) Why spikes? Hebbian learning and retrieval of time-resolved excitation patterns. Biol Cybern 69: 503-515

Gerstner W, Kempter R, van Hemmen JL, Wagner H (1996) A neuronal learning rule for sub-millisecond temporal coding. Nature 383: 76-78

Gerstner W, Kempter R, van Hemmen J, Wagner H (1997) A developmental learning rule for coincidence tuning in the barn owl auditory system. In: Bower J (ed) Computational neuroscience: trends in research 1997. Plenum, New York, pp 665669

Gerstner W, Kempter R, van Hemmen JL (1998) Hebbian learning of pulse timing in the barn owl auditory system. In: Maass W, Bishop CM (eds) Pulsed neural networks. MIT Press, Cambridge, Mass., pp 353-377

Grossberg S (1987) The adaptive brain. Elsevier, Amsterdam

Gustafsson B, Wigstrom H, Abraham WC, Huang Y-Y (1987) Long-term potentiation in the hippocampus using depolarizing current pulses as the conditioning stimulus. J Neurosci 7: 774780

Hebb DO (1949) The organization of behavior. Wiley, New York Hertz J, Krogh A, Palmer RG (1991) Introduction to the theory of neural computation. Addison-Wesley, Redwood City, Calif.

Herz AVM, Sulzer B, Kühn R, van Hemmen JL (1989) Hebbian learning reconsidered: representation of static and dynamic objects in associative neural nets. Biol Cybern 60: 457-467

James W (1890) Psychology (briefer course). Holt, New York, Chap 16

Kempter R, Gerstner W, and van Hemmen JL (1999) Hebbian learning and spiking neurons. Phys Rev E 59: 4498-4514

Kempter R, Gerstner W, van Hemmen JL (2001) Intrinsic stabilization of output rates by spike-based Hebbian learning. Neural Comput 13: 2709-2741

Kistler WM (2002) Spike-timing-dependent plasticity: a phenomenological framework. Biol Cybern 87: 416-427

Kistler WM, van Hemmen JL (2000) Modeling synaptic plasticity in conjunction with the timing of pre- and postsynaptic potentials. Neural Comput 12: 385-405

Kistler WM, Gerstner W, van Hemmen JL (1997) Reduction of Hodgkin-Huxley equations to a single-variable threshold model. Neural Comput 9: 1015-1045

Kohonen T (1984) Self-organization and associative memory. Springer, Berlin Heidelberg New York

Lapicque L (1907) Recherches quantitatives sur l'excitation electrique des nerfs traitée comme une polarization. J Physiol Pathol Gen 9: 620-635

Levy WB, Stewart D (1983) Temporal contiguity requirements for long-term associative potentiation/depression in hippocampus. Neurosci 8: 791-797
Magee JC, Johnston D (1997) A synaptically controlled associative signal for Hebbian plasticity in hippocampal neurons. Science 275: 209-213

Markram H, Lübke J, Frotscher M, Sakmann B (1997) Regulation of synaptic efficacy by coincidence of postysnaptic APs and EPSPs. Science 275: 213-215

Miller KD, MacKay DJC (1994) The role of constraints in Hebbian learning. Neural Comput 6: 100-126

Minai A, Levy WB (1993) Sequence learning in a single trial. In: Proceedings of the INNS World Congress on Neural Networks II, Portland, Oregon, 11-15 July, pp 505-508

Oja E (1982) A simplified neuron model as a principal component analyzer. J Math Biol 15: 267-273

Roberts P (1999) Computational consequences of temporally asymmetric learning rules: I. Differential hebbian learning. J Comput Neurosci 7: 235-246

Roberts P (2000) Dynamics of temporal learning rules. Phys Rev E 62: 4077-4082

Roberts P, Bell C (2000) Computational consequences of temporally asymmetric learning rules: II. Sensory image cancellation. J Comput Neurosci 9: 67-83

Rossum MCW van, Bi GQ, Turrigiano GG (2000) Stable Hebbian learning from spike timing-dependent plasticity. J Neurosci 20 : $8812-8821$

Rubin J, Lee DD, Sompolinsky H (2001) Equilibrium properties of temporally asymmetric Hebbian plasticity. Phys Rev Lett 86: 364-367

Sejnowski TJ, Tesauro G (1989) The Hebb rule for synaptic plasticity: algorithms and implementations. In: Byrne JH, Berry WO (eds) Neural models of plasticity. Academic, San Diego, pp 94-103

Senn W, Tsodyks M, Markram H (2001) An algorithm for modifying neurotransmitter release probability based on pre- and postsynaptic spike timing. Neural Comput 13: 35-67

Senn W, Schneider M, Ruf B (2002) Activity-dependent development of axonal and dendritic delays or, why synaptic transmission should be unreliable. Neural Comput 14: 583-619

Shouval HZ, Bear MF, Cooper LN (2002) A unified model of NMDA receptor dependent bidirectional synaptic plasticity. Proc Natl Acad Sci USA 99: 10831-10836

Song S, Miller K, Abbott L (2000) Competitive Hebbian learning through spike-time-dependent synaptic plasticity. Nat Neurosci 3: $919-926$

Stein RB (1965) A theoretical analysis of neuronal variability. Biophys J 5: 173-194

Xie X, Seung S (2000) Spike-based learning rules and stabilization of persistent neural activity. In: Solla SA, Leen T, Müller K-R (eds) Advances in neural information processing systems 12. MIT Press, Cambridge, Mass., pp 199-205

Zhang L, Tao H, Holt C, Harris WA, Poo M-M (1998) A critical window for cooperation and competition among developing retinotectal synapses. Nature 395: 37-44 